\title{
¿TENEMOS UNA ESTRATEGIA NACIONAL FRENTE A LA VIOLENCIA DE GÉNERO? ALGUNAS IDEAS PARA SU CONSTRUCCIÓN A PROPÓSITO DEL DECRETO LEGISLATIVO N$^{\circ} 1368 .{ }^{1}$
}

\author{
DO WE HAVE A NATIONAL STRATEGY AGAINST GENDER VIOLENCE.? \\ IDEAS FOR ITS CONTRUCTION WITH REGARD TO D. LEGISLATIVE N 1368
}

Christian Hernández Alarcón*

\section{Resumen}

En el presente texto, se cuestiona la forma en la que el Estado está luchando frente a la violencia que afecta a la mujer y a los integrantes del grupo familiar, la ausencia de una estrategia clara expresada en las marchas y contra marchas que han significado los cambios normativos recientes, dan cuenta de una intención de mejora que no se logra consolidar a pesar del tiempo transcurrido desde la entrada en vigencia de la Ley.

Palabras clave: violencia, mujer.

\begin{abstract}
:
This text questions the way in whitch the state is fighting against violence affecting women and members of the family group. The absence of a clear strategy as illustrated by recent advances and setbacks of regulatory amendments, show an intention of improvement that fails to consolidate despite the time elapsed since the entry into force of the law.

In this context, the enactment of Legislative Decree 1368, which creates the Specialized Justice System to address some violence situations, represents a challenge that may serve to achieve the necessary interagency articulation and affectively wage this fight but this is still early considering that we do not even have the basic protocol for joint action, articulation instrument provided by Law 30364.
\end{abstract}

Keywords: violence, women.

Ponencia presentada en el Pre Consejo Mundial de los Derechos de la Niñez y de la Adolescencia, organizado por el Colegio de Abogados de Lima y realizado el 7 y 8 de Setiembre de 2018.

1 Juez Superior de la Corte de Ventanilla, integrante de la Comisión de Género del Poder Judicial, profesor de Derecho de Familia de la PUCP y de la Academia de la Magistratura. 


\section{INTRODUCCIÓN}

La emisión del Decreto Legislativo 1368, que crea el Sistema Especializado de Justicia, para atender algunas situaciones de violencia, representa un reto que puede servir para concretar la necesaria articulación intersectorial necesaria para la efectividad de esta lucha pero aún incipiente teniendo en cuenta que no tenemos aún ni siquiera el protocolo base de actuación conjunta, instrumento de articulación previsto por la Ley 30364.

\section{PUNTO DE PARTIDA.- JUSTIFICACIÓN DE LA CREACIÓN DE UN SISTEMA DE PROTECCIÓN Y SANCIÓN INTEGRADO Y ESPECIALIZADO.}

La erradicación de la Violencia de género y la Violencia que afecta a los diversos miembros del grupo familiar, no depende exclusivamente de leyes; hace falta un esfuerzo conjunto del Estado y la Sociedad Civil, en el que el primero debe asumir un rol articulador y promotor, mientras que a la segunda le corresponde manifestar su cooperación con la administración de justicia y su no tolerancia frente a la violencia familiar.

Lamentablemente el Estado no ha cumplido su rol organizador de los servicios y mucho menos de construir una ruta articulada que brinde a las víctimas de violencia asistencia (psicológica, social, legal etc.) que contribuya con el objetivo de brindarle una protección integral de sus derechos, en especial su derecho a la tutela procesal efectiva (acceso a la justicia, debido proceso y efectividad de las decisiones)

En este contexto, la configuración normativa ha previsto desde hace mucho tiempo la necesidad de esta articulación, por lo que se empezó primero con la creación de una comisión multisectorial de alto nivel y el establecimiento de un plan de acción, intención que ha sido fortalecida con la Ley $\mathrm{N}^{\circ} 30364$, la misma que en su Título IV, ha previsto de modo expreso la creación de un Sistema nacional para la prevención, sanción y erradicación de la violencia contra la mujeres y los integrantes del grupo familiar, el cual conforme lo prevé el Artículo 33, tiene como funciones planificar, organizar y ejecutar acciones articuladas integradas y complementarias en la prevención, atención, protección y reparación de víctimas, la sanción y reeducación del agresor a efectos de lograr la erradicación de la violencia contra las mujeres y los integrantes del grupo familiar. Sistema que comprende una Comisión Multisectorial de alto nivel (Art. 35) un grupo de trabajo nacional, instancias regionales, provinciales y distritales de concertación, así como instrumentos y mecanismos de articulación, la cual ha considerado de modo expreso como instrumentos y mecanismos de articulación la construcción de un protocolo base de actuación conjunta, el registro único de 
víctimas y agresores, el observatorio de violencia, y el centro de altos estudios, muchos de los cuales aún se encuentran en pleno proceso de construcción a pesar del tiempo transcurrido. Lo que evidencia, una seria incapacidad para articular intersectorialmente de modo efectivo

En este contexto, mediante, Decreto Legislativo, 1368, sin que esta norma sea propuesta por el grupo de trabajo nacional, es decir de un instrumento de articulación ni tampoco el resultado de la deliberación del congreso, se crea el Sistema Nacional Especializado de Justicia para la protección y sanción de la violencia contra las mujeres, e integrantes del grupo familiar, lo que en la práctica es un "subsistema de justicia" para atender algunas situaciones de violencia ${ }^{2}$, las demás situaciones, no elegidas permanecerán a cargo del sistema ordinario.

La implementación y funcionamiento de este subsistema, implica la articulación de modo progresivo y especializado en su actuación de las entidades del sistema de justicia (Poder Judicial, Ministerio Público, Policía Nacional, Ministerio de Justicia, Ministerio de la Mujer) focalizando su intervención en la protección y la sanción.

Sin embargo, la protección y la sanción, que corresponde al sistema de justicia, ya sea ordinario o especializado, como es el caso del sub sistema, no deja de estar vinculado con otras acciones que dependen del sistema nacional en su conjunto. Situación que hace urgente establecer mecanismos de coordinación, sin los cuales no puede esperarse efectividad en su actuación.

\begin{tabular}{|l|l|}
\hline $\begin{array}{l}\text { El sistema y subsistema articulan de acuerdo a } \\
\text { la ley: }\end{array}$ & $\begin{array}{l}\text { Las actuaciones de justicia están vinculadas y deben } \\
\text { estar dentro del subsistema }\end{array}$ \\
\hline Prevención & Primaria y secundaria \\
\hline Atención & $\begin{array}{l}\text { Derecho a la información, defensa legal y atención } \\
\text { integral para el acceso a la justicia. }\end{array}$ \\
\hline Protección & $\mathrm{X}$ \\
\hline Sanción & $\mathrm{X}$ \\
\hline Reparación de víctimas & $\begin{array}{l}\text { Es derecho de la víctima, implica una restitución de su } \\
\text { derecho y al mismo tiempo es considerado medida de } \\
\text { protección de tutela estatal. }\end{array}$ \\
\hline Reeducación de agresores & $\begin{array}{l}\text { Es dispuesto por orden judicial y debe ser supervisado } \\
\text { en su cumplimiento. }\end{array}$ \\
\hline
\end{tabular}

Art. 3 del Decreto Legislativo $\mathrm{N}^{\circ} 1368$, señala que se debe aplicar en los casos de Feminicidio, lesiones previstas en los artículos 121-B, 122, 122 B en concordancia con el Artículo 124 -B, Violación sexual, previstos en los Artículos 172, 173, 173-A y 174 y sus formas agravadas comprendidas en el Artículo 177 del Código Penal cuando la víctima es agredida por su condición de tal o son niños, niñas o adolescentes y actos contra el pudor previsto en el Artículo 176-A del Código Penal . 
La protección y sanción efectiva depende del acceso a la justicia, y éste de mecanismos de detección precoz de violencia y canales y servicios de atención. Del mismo modo, las decisiones de protección y sanción, deben cumplirse efectuándose un seguimiento de las mismas, cuyo éxito, trasciende el funcionamiento del propio sistema de justicia.

Para ello, debe haberse adoptado de modo previo una ruta de atención, organizándose la oferta de servicios en forma complementaria, integrada y articulada, y luego ésta debe desplegarse en la realidad concreta.

Nada esto pasa, si previamente no construimos un sistema que pase del papel a la realidad. En las siguientes líneas, nos empeñaremos en explicar los elementos componentes de un sistema, y daremos algunas ideas para ir del dicho al hecho.

\section{DEBEMOS ENTENDER PRIMERO QUÉ SIGNIFICA UN SISTEMA DE PROTECCIÓN}

La ley crea un marco, importante pero insuficiente. En este contexto, el sistema es la única garantía de que estas previsiones legales se lleven a la práctica, sistema que debe ser la reunión armoniosa e integrada, de todos los elementos y recursos necesarios diseñados para efectivizar que los servicios ofrecidos por las diferentes instituciones funcionen de modo complementario y articulado, para asegurar el derecho de la víctima a la tutela jurisdiccional efectiva, por medio de la tutela protectora, y que respondan de modo efectivo al agresor, con una sanción la cual responde a la legítima aspiración de justicia de la víctima y al mismo tiempo, representa un mensaje claro a la sociedad de no tolerancia frente a la violencia.

La razón de la necesidad de este sistema es que tanto la impunidad como las respuestas no efectivas, generan violencia. De allí, su necesidad de que no sea impuesto sino gestado es decir, construido cooperativamente con el objeto de congregar a todos los actores involucrados, y determinar su nivel de compromiso y actuación concreta, por lo que esta articulación se tenía que haber realizado desde el protocolo base de actuación conjunta, y efectivizado la construcción de protocolos locales de actuación, situación que no se ha efectuado y ojalá con esta norma, se efectivice al fin.

El Sistema debe tener como punto de partida todas las investigaciones disponibles sobre el tema, pues en otras realidades ya se han construido esfuerzos en este sentido. Asimismo, debe retroalimentarse con el análisis de los derechos y necesidades de las víctimas, Sin embargo la clave se encuentra en el análisis concreto de las ofertas de servicios que ofrecen las instituciones y las organizaciones locales, con el fin de establecer una ruta de atención integrada y complementaria hacia la protección y sanción efectiva, evitando, situaciones 
críticas: ausencia de oferta, dispersión y superposición de servicios, que generan en las victimas desánimo, deserción, indefensión en la tutela de sus derechos, medidas de protección inadecuadas, no ejecutadas adecuadamente y sin seguimiento efectivo, lo que aunada a una falta de sanciones efectivas, genera una sensación colectiva de inseguridad e impunidad.

\section{LA NECESARIA CONSTRUCCIÓN DE UNA SOLA VISIÓN DE CONJUNTO SOBRE LA VIOLENCIA DE GÉNERO PARA LA DETERMINACIÓN DE OBJETIVOS DEL SISTEMA Y DE SUS PRIORIDADES DE ATENCIÓN}

Es necesaria una sola visión de conjunto sobre la violencia de género que permita a todos los elementos del sistema intervinientes, tener los mismos objetivos y las mismas prioridades de atención.

En este sentido, consideramos prioritario:

Dar seguridad a la víctima, previa valoración adecuada del riesgo, situación que implica información para ella de todas las opciones a su disposición, mecanismos de priorización de atención de casos y establecimiento de rutas diferenciadas en función del riesgo. Asegurando una medida oportuna, adecuada integral, que además se ejecute y tenga mecanismos de seguimiento. Al respecto, no hemos medido el impacto ni validez de las fichas de valoración, ni tampoco si pueden hacerse valoraciones de riesgo cualitativas, como las que se pretendieron implementar desde el Ministerio Público, el 2009, con la cooperación e Eurosocial JUSTICIA. Hay que analizar todas las posibilidades, incluso habrá que hacerse cargo de los mecanismos que deben implementarse si ella deserta, para que pueda retomar el caso o volver a denunciar.

Efectuar una investigación con debida diligencia que evite la impunidad de los agresores y ponga en claro que no puede burlase del sistema. Al mismo tiempo, debe contar con opciones de cambio de conducta.

Todos los servicios deben de estar coordinados para complementarse sin repetir innecesariamente esfuerzos. Del mismo modo, debe de potenciarse una intervención interdisciplinaria que permita describir las necesidades individuales de las víctimas, agresores, de sus hijos es decir de las víctimas directas e indirectas.

La oferta de servicios y la ruta de atención debe diferenciar por ejemplo zonas rurales donde la vulnerabilidad e interseccionalidad se manifiestan de modo distinto. 


\section{UNA LEGISLACIÓN ADECUADA.}

El mayor defecto que se está cometiendo en la lucha contra la violencia en nuestra ley es que dicha ley y sus modificatorias, no responden a una visión de conjunto (Estado-Sociedad) sobre la violencia, ni tampoco del establecimiento de un Sistema de Protección frente a ella, con un plan de acción específico y rutas de atención diferenciadas y adaptadas en los distintos entornos locales.

En este sentido, teniendo en cuenta que si bien el aspecto legal es simplemente una herramienta más de todo el Sistema, consideramos que debe tener las siguientes características:

a) La ley debe de responder a un Sistema, es decir a sus objetivos y prioridades.

b) La ley debe tener vocación de permanencia no puede ser modificada a cada rato, y por tanto debe tener mecanismos de seguimiento que permitan retroalimentar el sistema y promover mejoras, pero lo que no puede suceder es que se hagan modificaciones irresponsables que no respondan a estos objetivos de mejora, como por ejemplo que sólo impliquen disminución de plazos sin una base fáctica que sustente estas modificaciones, o que se cambie el enfoque de una ruta penal y civil paralela, luego a la integración de la tutela con la 30364, y recientemente a la separación de ambas. Estas contradicciones, dan cuenta de una falla sistémica: No hemos reflexionado suficientemente para determinar que debemos hacer. Por otro lado, estos cambios repentinos, y no reflexivos, generan confusión en los operadores y se constituyen en un obstáculo para la articulación del sistema pues no son el resultado de su retroalimentación y muchas veces se dan especialmente en el ámbito de justicia de espaldas a los propios operadores.

c) La ley debe de responder a las necesidades de las víctimas y dejando de lado las rutas complicadas como por ejemplo, la división de la defensa respecto de alimentos a cargo del ministerio de justicia y violencia a cargo de los centros de emergencia mujer. Frente a ello, proponemos una ruta simple, no engorrosa, la cual parte del rompimiento de los compartimentos estancos del derecho de familia y penal, por lo que si no hay una sola vía de protección y penal, debe haber suficientes mecanismos de coordinación y contacto que impidan que la víctima sea revictimizada por tener que tocar puertas distintas las cuales, además de ser obligatorias no se encuentran conectadas. La víctima debe tener una ventanilla única, una sola ruta sin perjuicio de que en su interior la administración deba tener diversas conexiones imbricadas para 
responderle. Proceso sencillo, expeditivo no dilatorio. Las vías superpuestas no dan buenos resultados porque confunde a las víctimas que no tienen una idea clara de cómo funciona el sistema, ni saben, la ruta a seguir.

\section{FORMACIÓN DE REDES DE COOPERACIÓN INTERINSTITU- CIONAL Y EL FORTALECIMIENTO DE LAS EXISTENTES}

Es fundamental la formación de Redes de cooperación interinstitucional y el fortalecimiento de las existentes a fin de integrar la intervención fiscal, judicial, con servicios que atiendan integralmente a las víctimas y agresores. Debe saberse donde están los servicios y como se pueden complementar.

Debe de establecerse, asimismo, conexión entre el sistema que protege y sanciona con las redes vinculadas a la prevención entre las instituciones barriales y vecinales con la finalidad de detectar e intervenir en forma inmediata frente a una situación de violencia, así como de canalizar a las víctimas hacia las vías más favorables de acuerdo a su caso.

\section{UN TRABAJO COORDINADO}

Un trabajo coordinado implica claridad en las responsabilidades y competencias de cada cual, integración de procesos para la atención, estandarización de actuaciones, mecanismos de derivación y seguimiento.

En este sentido, debe evitarse las sucesivas investigaciones que se dan en muchas instancias, con repetición innecesaria de los mismos medios probatorios y de las mismas diligencias. Las actuaciones deben servir para todas las instancias, y todas deben ser retroalimentadas por la información generada en todas las instancias e instituciones.

¿Pueden los jueces ver los reconocimientos médico legales que ya se han actuado, así como los propios fiscales sin necesidad de remitir oficios? No pueden, pero deben. La información y su calidad incide en la calidad de la decisión, la generación de información debe enriquecer la calidad de todos los actores que están encargados de tomar decisiones. La implementación de esta medida no necesita modificación normativa, ni nuevo sistema informático, ni interoperabilidad, solo coordinación concreta plasmada en un protocolo. Ente Coordinador de todo el Sistema que planifique el trabajo, planteándose metas concretas, verifique los resultados, evalúe anualmente la forma como intervienen las diferentes instancias del sistema, que analice los logros obtenidos, promueva el debate y esté abierto a las propuestas de modificatorias retroalimentando el sistema. 


\section{RECURSOS ECONÓMICOS Y HUMANOS}

No podemos pensar en el desarrollo económico, si nos olvidamos del desarrollo social o humano, dentro de éste, debe garantizarse una adecuada participación de la mujer - invertir en las personas promocionando la equidad entre los géneros es la base para el crecimiento económico y un desarrollo sostenido. Es imprescindible "Transformar la relación entre el hombre y la mujer, basándose en la igualdad para lograr un desarrollo sostenible del ser humano"3.

En este sentido, siendo la violencia un problema de desarrollo, de la lucha contra ella depende un mejor nivel de vida de la familia y por ende de la sociedad entera. Un Sistema de Protección, debe también de contar con un presupuesto económico, sin él no podría realizarse todos los objetivos que se plantea. Leyes sin presupuesto son leyes sin posibilidad de implementación. Estados que no invierten en leyes que aseguran ejercicio de derechos son estados que no garantizan el ejercicio de estos derechos.

Estos recursos económicos, deben garantizar recursos humanos especializados: hombres y mujeres que habiendo roto con la espiral de violencia en sus propias vidas y familias, se encuentren capacitados y comprometidos con la lucha contra la violencia, facilitando el acceso a la justicia, a las pruebas y su aseguramiento

Se necesitan entonces, personas que no se conviertan por su actuación revictimizante, en una razón más para la deserción de las víctimas.

Herramienta humana fundamental del Sistema es el juez, quien debe de entender que no es un mero aplicador de la ley, sino un creador de Derecho. Correspondiéndole la habilidad, la intuición y la imaginación creadora necesaria para extraer del texto abstracto y genérico de la ley un mandato legal específico para el caso que resuelve. (Monroy Galvez, Juan, 1995, p.66) Por esta razón, no debe ser dejado de lado en las modificaciones normativas, ni tampoco en las instancias de articulación del sistema de justicia.

\section{ARTICULACIÓN DE LAS ACCIONES DEL SISTEMA EN UN PLAN DE ACCIÓN CON NIVELES DE INTERVENCIÓN}

Un Sistema de Protección que pretenda ser efectivo, debe contar con la participación de todos los agentes involucrados, integrados en su actuación por medio de un protocolo que permita articular la intervención estatal, para no producir dobles intervenciones e interferencias innecesarias.

Objetivo de la Plataforma de las Mujeres de la Conferencia de Beijin, p. 5. 
Debe fijarse por ello, niveles de intervención, para que cada elemento del sistema tenga perfectamente delimitada su competencia, pues muchas veces pueden intervenir más de una institución pero debe saberse quien interviene en el caso concreto para hacerlo de modo adecuado.

Es importante, delimitar niveles de intervención:

1. Intervención temprana, la que incluye la detección de los servicios de educación y salud desde donde debe derivarse el caso teniéndose en cuenta la valoración del riesgo y las posibilidades de derivación a las instancias de investigación y decisión, en qué caso (identificación de signos), qué orientación deben dar a las víctimas, y cómo deben derivar debe ser definido.

2. Intervención en crisis, la que implica la denuncia efectuada por la víctima a la policía, u otra institución que implica la búsqueda de una decisión de protección o restrictiva de derechos. Aquí las víctimas deben recibir protección inmediata y efectiva que garantice su seguridad, el agresor al mismo tiempo debe recibir un mensaje claro respecto a la no tolerancia de la violencia. Consideramos situaciones de crisis de especial atención:

a) Delitos cometidos en agravio de la víctima que merezcan combinación de medidas. Por ejemplo: el agresor no tiene prisión preventiva, y la víctima no puede regresar a su casa, por lo que además de sacar al agresor de la casa se necesitan medidas cautelares de tenencia y alimentos.

b) La valoración del riesgo es alta y por tanto la orden de alejamiento o salida del agresor es la indicada, así el hecho no sea considerado un delito grave de acuerdo al sistema penal o incluso no sea delito.

c) Situación de intervención en flagrancia, la cual puede configurarse de dos formas: el agresor se encuentra en flagrancia por haber efectuado un acto de violencia y ha sido detenido en dicha circunstancia o en momento inmediato posterior, o el agresor sin que necesariamente haya efectuado un nuevo acto de violencia, ha quebrantado la medida de protección dictada con anterioridad. En ambos casos, debe ser detenido, y con independencia de la necesidad de revisar la medida de protección dictada para que de ser el caso sea intensificada, debe procederse conforme a las reglas de proceso inmediato y ser sancionado por la conducta cometida. La no sanción inmediata en las situaciones de flagrancia, es una práctica que legitima las situaciones de violencia y debe ser desterrada. 
Intervención de Seguimiento de corta duración, de duración media y prolongado, luego de la adopción de las decisiones de protección en situación de crisis, debe efectuarse una prognosis de seguimiento del caso en función de la valoración del riesgo. Debe estandarizarse el tipo de acciones que deben efectuarse en cada tipo de seguimiento y los plazos. La valoración de riesgo en estos casos debe ser efectuada en forma periódica y de su resultado depende el que se terminen los mecanismos de seguimiento. Sin embargo, el sistema debe de entender que no puede asumir compromisos que superen sus fuerzas y recursos; por ello, debe valorar cuantitativamente y cualitativamente el riesgo para quedarse en seguimiento únicamente con aquellos casos que lo ameriten.

Para determinar la actuación específica de las instituciones involucradas dentro de cada nivel de intervención, es necesario saber cuáles son las acciones concretas que desarrollarán el Estado y la Sociedad Civil, los objetivos del Sistema y sus prioridades de atención.

\section{RECOMENDACIONES ESPECÍFICAS PARA LA IMPLEMEN- TACIÓN}

\subsection{Coordinación y Capacitación}

Es fundamental que el diseño e implementación requiera una coordinación no desligada de las necesidades de las víctimas y que recoja todos los aportes de la comunidad en la lucha contra la violencia.

Para la coordinación y capacitación debe funcionar adecuadamente la comisión de alto nivel y el grupo de trabajo nacional.

La falta de una adecuada capacitación es el resultado de una minimización de la labor de los operadores dedicados al abordaje de la violencia. Debe entenderse que, no es la ley el instrumento más importante del sistema, sino el elemento humano formado por los fiscales, jueces de familia y penales, de quienes depende en suma que todo el sistema funcione; sin embargo, sino están entrenados adecuadamente: por ejemplo, conoce la ley pero no la dinámica de la violencia; es decir, su formación debe ser interdisciplinaria.

Desde allí, debe gestarse las acreditaciones de los operadores, por medio de entrenamiento específico para el desarrollo de las competencias requeridas; como por ejemplo, para el tratamiento diferenciado de las víctimas y de los agresores. En el caso de los jueces y fiscales debe analizarse los logros en la detención de la violencia por medio del estudio de casos y su impacto concreto en las víctimas, con el fin de evaluar resultados, 
construyéndose un manual de procedimientos para actuar investigar y proteger con perspectiva de género.

Asimismo, para la construcción y consolidación de una visión de conjunto, las capacitaciones deben ser intersectoriales; es decir, de todos los operadores comprometidos en la intervención.

Del mismo modo, las campañas de difusión deben estar dirigidas a dar mensajes claros a las víctimas sobre las posibilidades del sistema en el acceso a la justicia y los servicios disponibles.

\subsection{Garantizar la defensa integral de las víctimas}

Propiciar que los operadores del estado tengan una buena relación con las víctimas para evitar su deserción implica mucho más que mejorar las competencias en atención; es actuar de modo objetivo y concreto con actos que demuestren un genuino interés por su situación, acompañarla, y proveerle asistencia integral (legal, psicológica y social).

Los servicios deben complementarse para proveer una defensa técnica de calidad y una atención integral a la víctima quele permita dar una salida definitiva a sus problemas y evite el empeoramiento de su situación. Confianza, protección debe sentir la víctima para que el caso no termine generando impunidad.

\subsection{Evaluación y Retroalimentación del Sistema}

El Artículo 5 del Decreto Legislativo 1368, señala que las entidades que conforman el Sistema deben establecer de modo articulado, mecanismos de monitoreo y evaluación de su implementación y funcionamiento y que los resultados se informan anualmente ante la Comisión Multisectorial de Alto Nivel del Sistema Nacional para la Prevención, Sanción y Erradicación de la Violencia contra las Mujeres y los Integrantes del Grupo Familiar, a que se refiere la Ley № 30364, Ley para prevenir, sancionar y erradicar la violencia contra las mujeres y los integrantes del grupo familiar.

Consideramos que en el monitoreo y evaluación debe considerarse y valorarse:

a) Si los objetivos propuestos se han cumplido.

b) Si responde a las necesidades actuales y a las investigaciones que han medido su impacto. 
c) Sistematizar las buenas experiencias, medir su impacto, consolidarlas evaluarlas y promover su réplica.

d) Priorizar las soluciones que no impliquen modificación legal a los problemas detectados, para evitar modificar innecesariamente el marco legislativo, lo cual es una mala práctica de abordaje pues genera problemas de adaptación e inseguridad jurídica en todos los niveles.

\section{CONCLUSIONES}

Protección y sanción efectiva, impunidad e inercia determinan como abordamos en concreto la violencia. Eso no depende del diseño normativo, sino del modo en el que lo llevamos a la práctica.

La lucha contra la violencia implica una respuesta que no se puede efectuar en solitario desde una sola institución o trabajando de modo aislado. La articulación debe ser concreta, la ruta simple y clara. Del mismo modo que el mensaje dado a la sociedad, a las víctimas y a los agresores.

\section{REFERENCIAS}

Monroy Gálvez, Juan (1995). En Busca del Juez Peruano: Hacia una Verdadera reforma del Poder Judicial. Entrevista, Themis, Segunda Época, № 32 .

Decreto Legislativo $\mathrm{N}^{\circ} 1368$ - Decreto Legislativo que crea el Sistema Nacional Especializado de Justicia para la protección y sanción de la violencia contra las mujeres e integrantes del grupo familiar, publicado el 29 de julio de 2018 en el Diario Oficial "El Peruano".

Fecha de recepción : 31 de agosto de 2018

Fecha de aceptación : 25 de octubre 2018 